Revue Sciences/Lettres

Sciences / Lettres

1 | 2013

Transferts culturels

\title{
Enregistrer ou l'archivage de la mesure
}

Denis Beaudouin et Michel Laguës

\section{(2) OpenEdition}

Journals

Édition électronique

URL : http://journals.openedition.org/rsl/338

DOI : $10.4000 /$ rsl.338

ISSN : 2271-6246

\section{Éditeur}

Éditions Rue d'Ulm

Référence électronique

Denis Beaudouin et Michel Laguës, «Enregistrer ou l'archivage de la mesure », Revue Sciences/Lettres [En ligne], 1 | 2013, mis en ligne le 01 juin 2012, consulté le 01 mai 2019. URL : http:// journals.openedition.org/rsl/338; DOI : 10.4000/rsl.338

Ce document a été généré automatiquement le 1 mai 2019.

(c) Revue Sciences/Lettres 


\title{
Enregistrer ou l'archivage de la mesure
}

\author{
Denis Beaudouin et Michel Laguës
}

Depuis 3,5 milliards d'années, les organismes vivants conservent l'information génétique sur l'ADN, un système de stockage moléculaire comprenant aussi des procédures de lecture et de réplication.

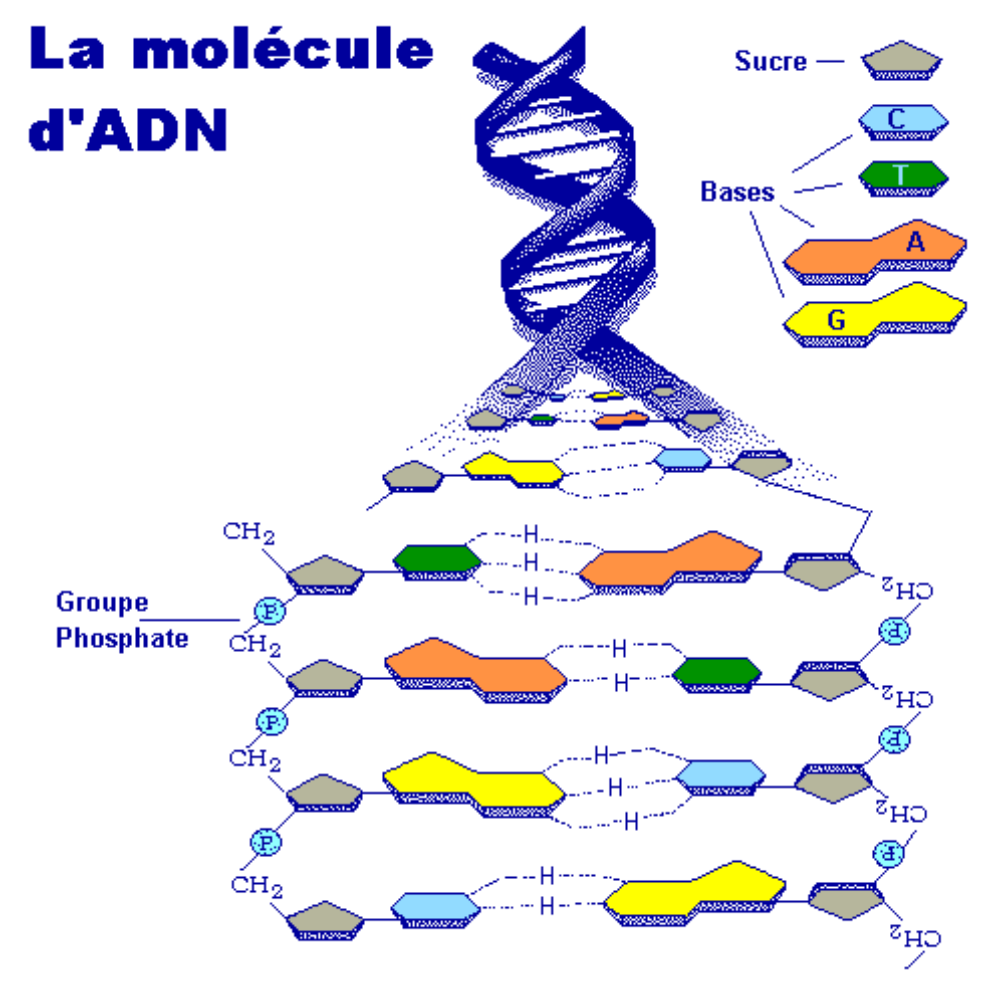

2 Puis, au fil de l'évolution du règne animal, le cerveau traite l'information "mémoire » dans un ensemble neuronal perfectionné, les mammifères supérieurs en devenant des champions. 
Il y a 70000 ans, l'homo sapiens grave ses premiers signes sur la pierre, puis dessine.

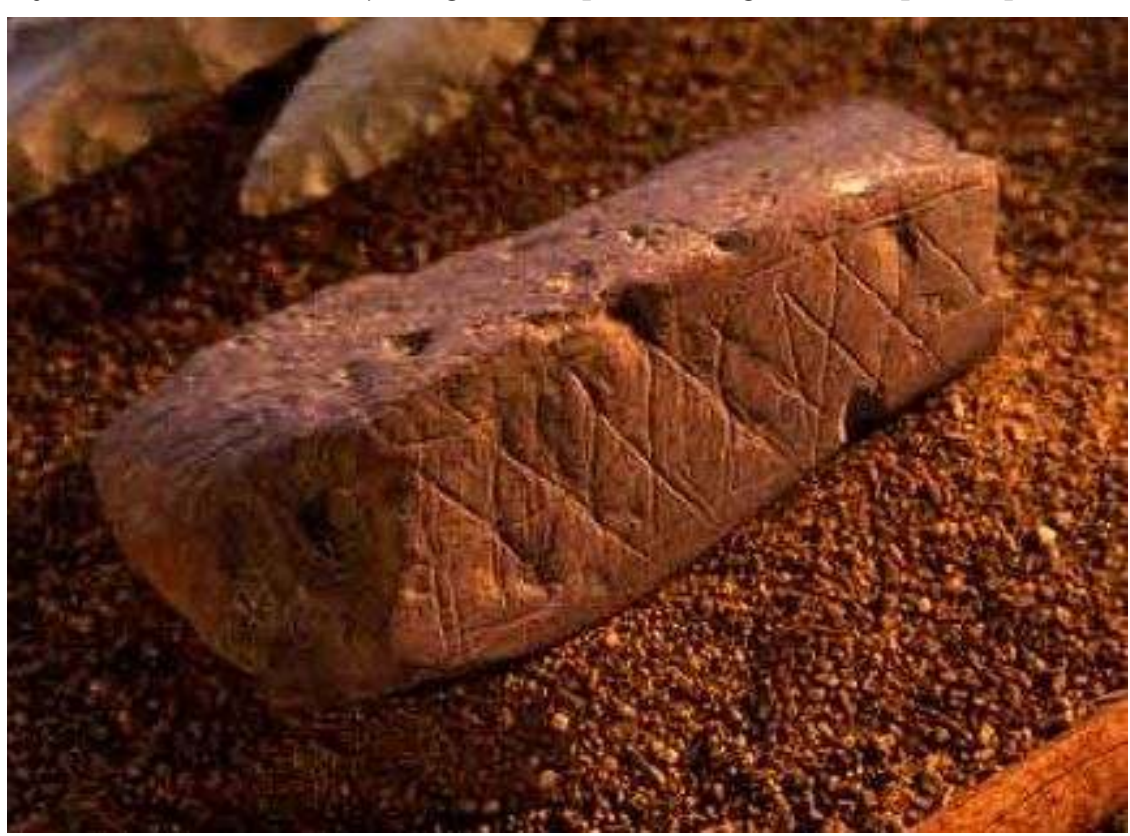

Francesco d'Errico, Chris Henshilwood et Peter Nilssen, « An engraved bone fragment from c. 70 000year-old Middle Stone Age levels at Blombos Cave, South Africa : implications for the origin of symbolism and language », Antiquity, 75, 2001, p. 309-318.

4 S'ensuivra l'écriture : l'inscription de mythes, d'observations et de mesures de toutes sortes sur une multiplicité de supports. C'est un accroissement fantastique des moyens de son cerveau pour mémoriser, traiter, communiquer, bref pour penser ${ }^{1}$ : l'homme s'est inventé des «mémoires externes ».

5 Depuis l'Antiquité, l'homme a imaginé toutes sortes de garde-temps pour saisir l'insaisissable, le moment fuyant. À la Renaissance, il invente d'ingénieuses horloges dont les mécanismes permettront ensuite, il y a moins de trois siècles, de concevoir des automates pour imiter la vie, et des mécaniques pour enregistrer les mesures, les phénomènes et le mouvement. Celles-ci vont progressivement se substituer à la main de l'homme pour inscrire l'information, démultipliant ses mémoires externes. 
Joueuse de tympanon

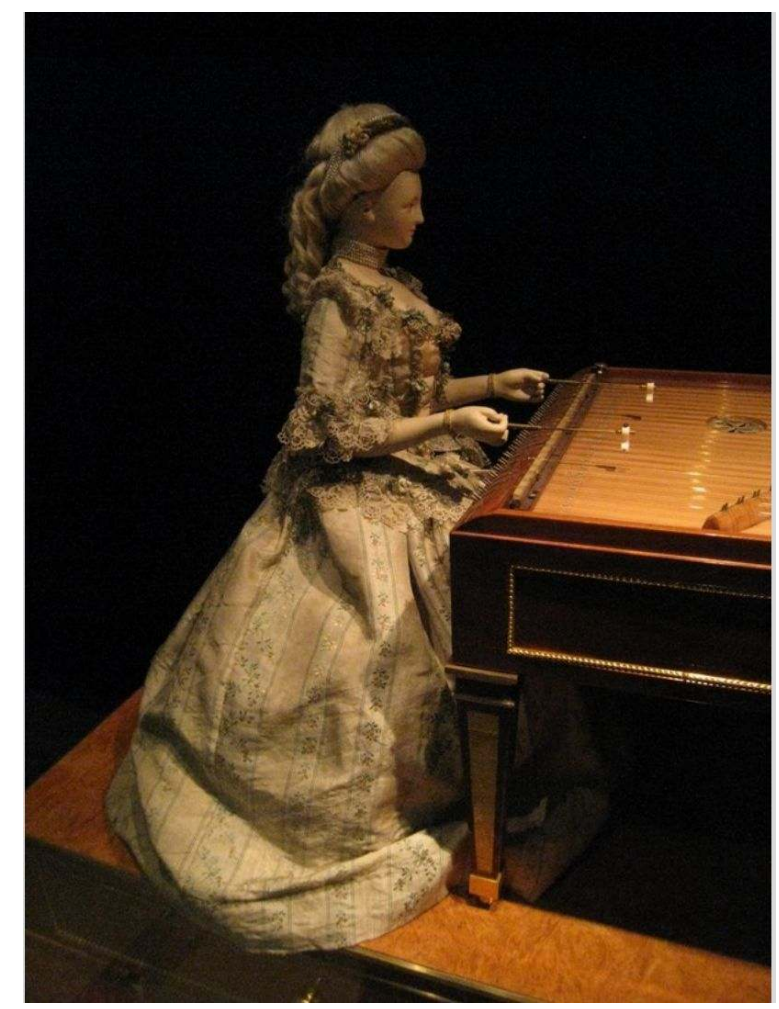

6 Comment ce processus a-t-il accéléré le progrès des sciences, et comment les découvertes scientifiques ont-elles perfectionné les enregistreurs? Comment, depuis moins de deux siècles, les systèmes enregistreurs ont-ils facilité l'accès à l'infiniment bref, à l'infiniment petit, à ce qui est caché aux sens de l'homme ? Où nous mène la progression vertigineuse des performances de l'enregistrement et du traitement de l'information? Voici quelquesunes des questions abordées dans ces lignes.

\section{Mécanique, argentique, électro-magnétisme, cathodique: des mariages féconds}

7 Quelques savants et artisans londoniens auraient réalisé des appareils météorologiques enregistreurs à la fin du $\mathrm{XVII}^{\mathrm{e}}$ siècle, mais il semble que le premier instrument ayant fonctionné soit l'Anémomètre de Pajot d'Ons-en-Bray de 1734, permettant d'enregistrer durant trente heures la force et la direction du vent ${ }^{2}$. 
L'anémomètre de Pajot d'Ons-en-Bray (1734)

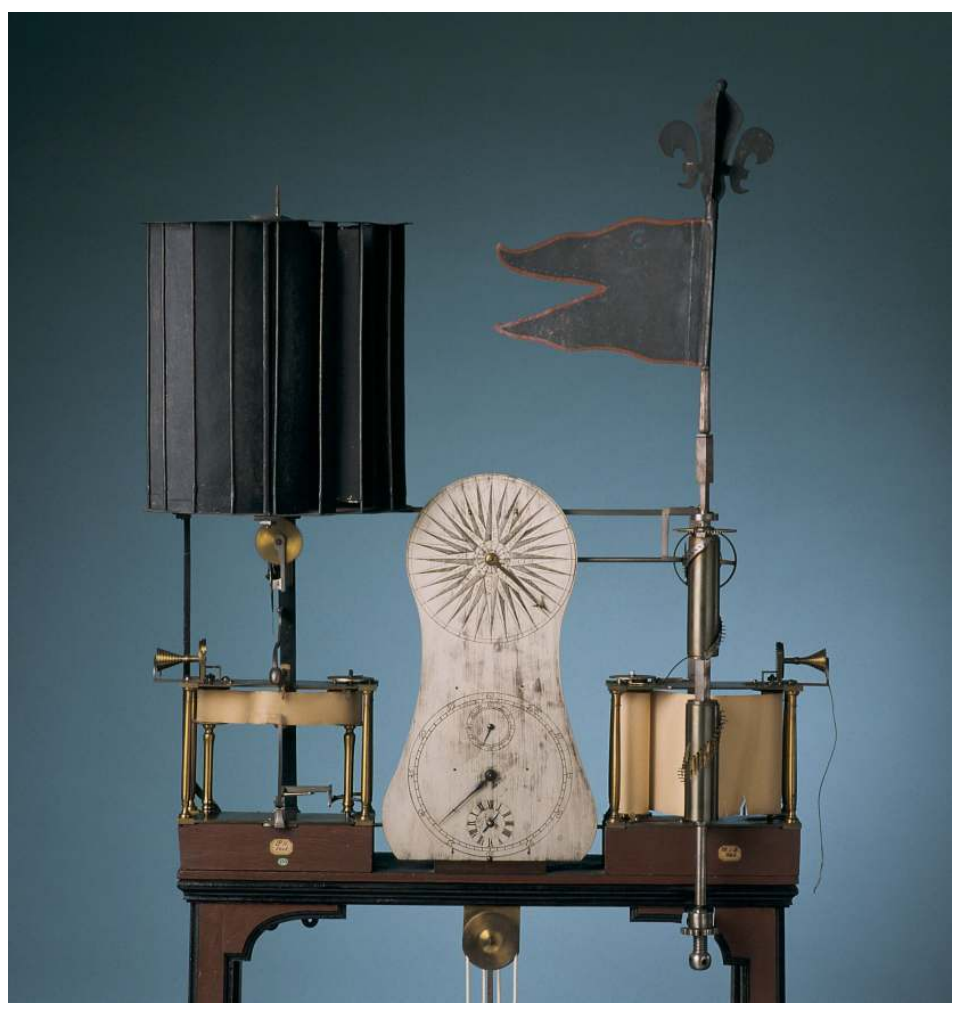

8 Le $\mathrm{XIX}^{\mathrm{e}}$ siècle voit apparaître un foisonnement de procédés d'enregistrement, parmi lesquels émergent quelques systèmes véritablement performants. Les premiers sont purement mécaniques :

- 1800 : l'indicateur de Watt permet d'enregistrer le travail des machines à vapeur, représenté par un diagramme de Clapeyron $(\mathrm{P}, \mathrm{V})^{3}$; 


\section{L'indicateur de Watt}

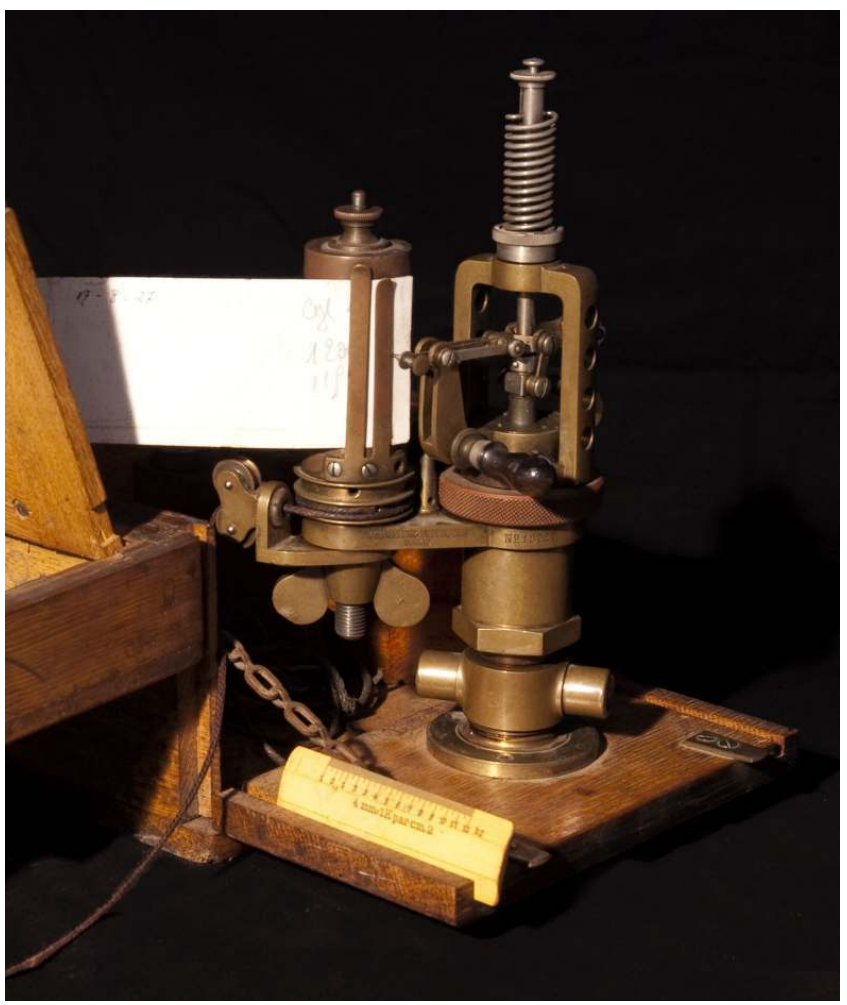

- 1857 : le Sphygmographe du Dr Marey ${ }^{4}$, avec lequel il met en évidence la diastole et la systole cardiaques ; 
Sphygmographe de Marey
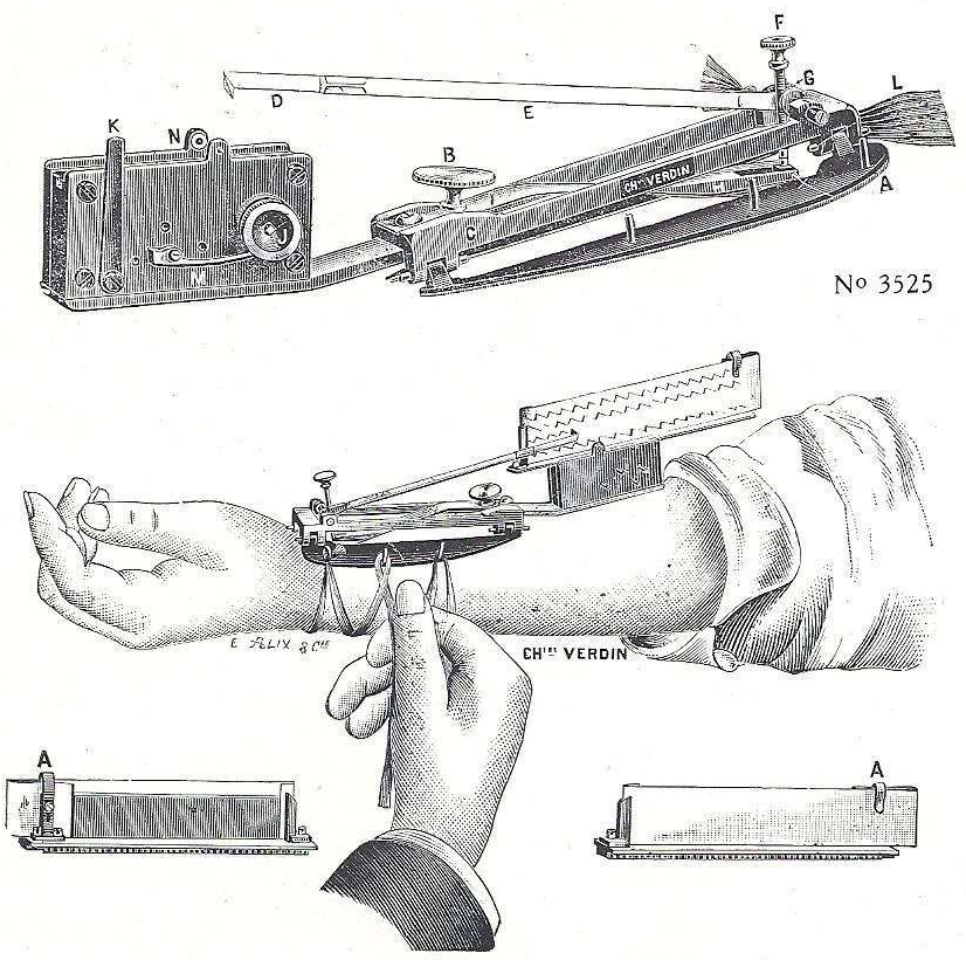

- 1857 : le phonautographe de Scott de Martinville, premiers essais d'enregistrement du son ${ }^{5}$.

\section{Phonautographe}

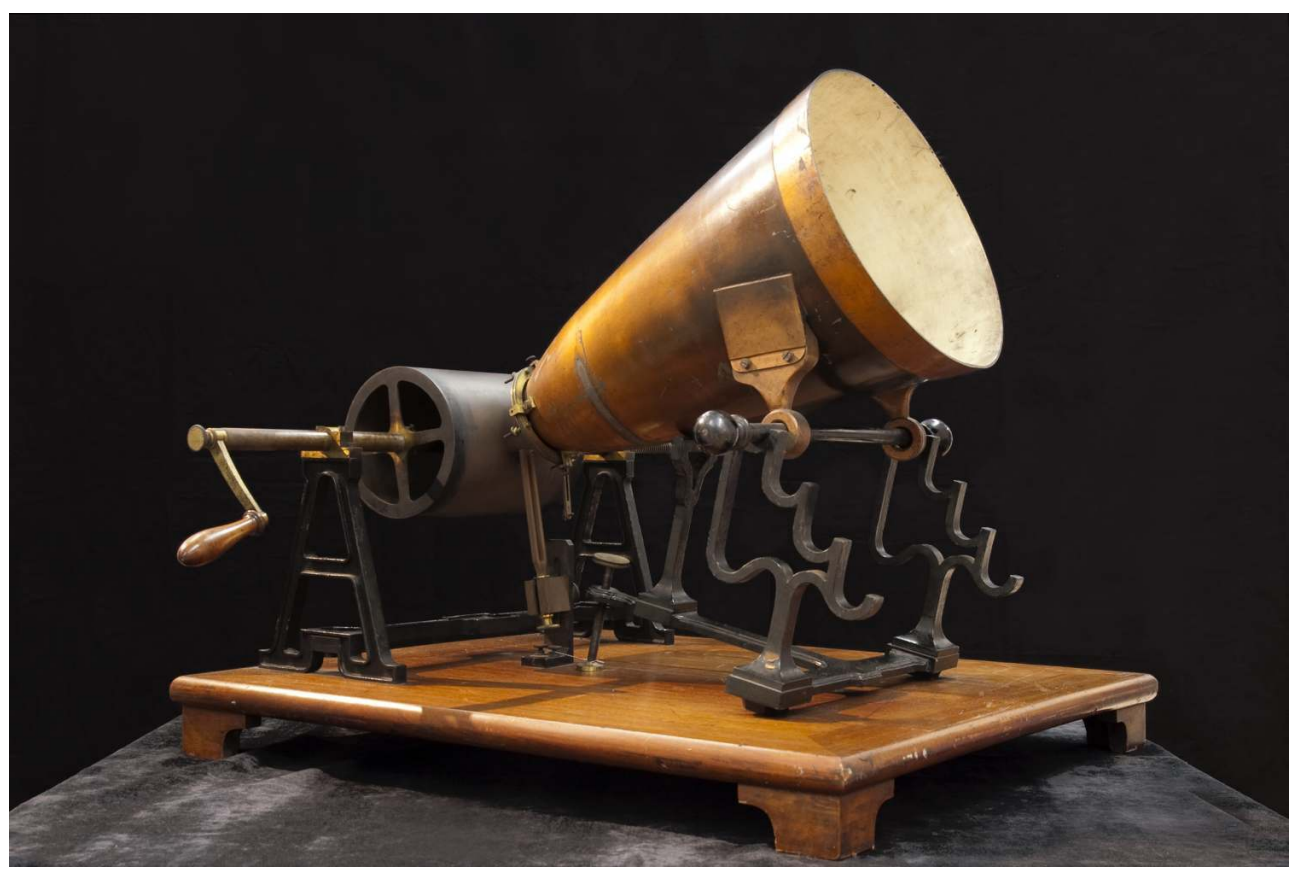

Dans les années 1870, la photographie argentique apporte à la mécanique un support d'enregistrement fiable, précis et durable. Mais le débat entre Janssen, physicien et tenant de la photographie, et Cornu, mathématicien et tenant de l'observation et du 
calcul, se poursuit quelques années avant que l'utilisation de la photographie dans la recherche ne soit définitivement acquise ${ }^{6}$.

10 Le sismographe de Palmieri ${ }^{7}$ de 1857 est sans doute le premier appareil utilisant les propriétés de l'électromagnétisme, récemment mises en évidence par Faraday.

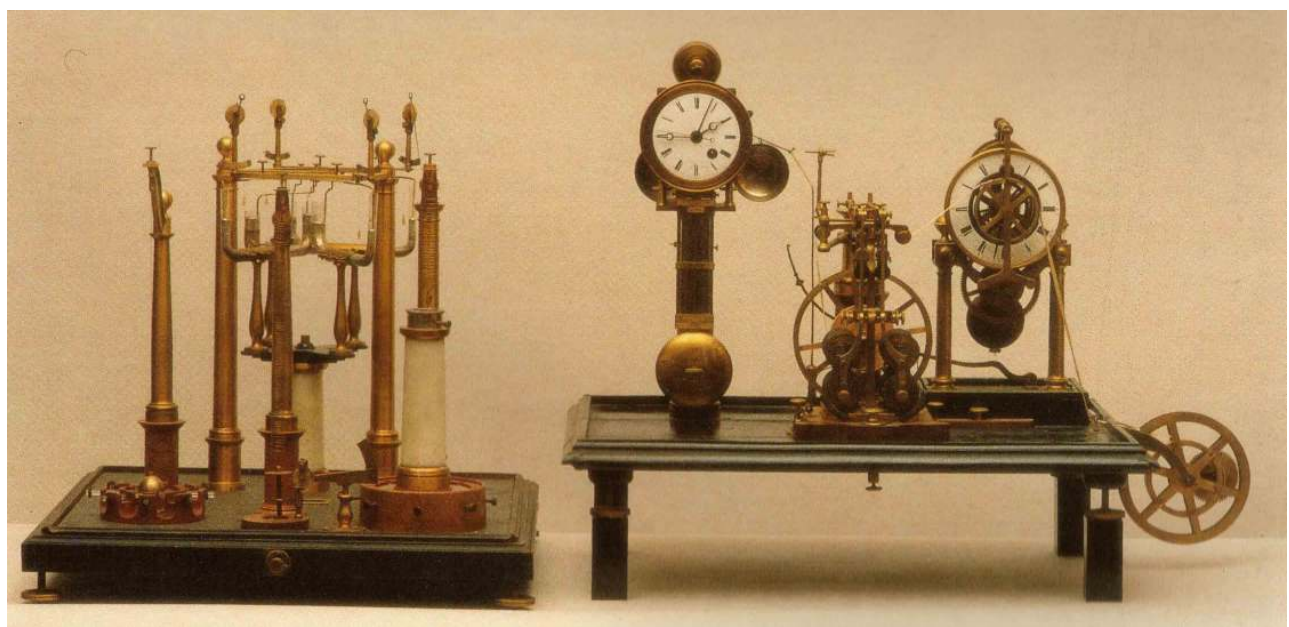

Sismographe de Palmieri

11 Il est suivi vingt ans plus tard par le wagon d'expériences de la $\mathrm{C}^{\mathrm{ie}}$ des Chemins de fer de l'Est, dont les enregistreurs sont construits par Desprez: c'est le premier enregistreur «embarqué $»^{8}$.

12 Mais c'est au tournant du $\mathrm{xx}^{\mathrm{e}}$ siècle que la course à la vitesse d'enregistrement s'accélère avec l'arrivée des oscillographes électromagnétiques performants de Henri Abraham (ENS) ${ }^{9}$ et d'André Blondel ${ }^{10}$ en France, de Duddell en Grande Bretagne, de Siemens en Allemagne, de Hotchkiss aux USA... On dépasse les fréquences d'enregistrement de $7 \mathrm{kHz}$ avant 1900 .

La communication de Blondel au Congrès des Électriciens tenu à Paris en $1900^{11}$ présente une belle synthèse des progrès récents. Elle esquisse les promesses de la découverte par Ferdinand Braun, en 1897, de la déviation des rayons cathodiques par les champs magnétiques et électriques.

Tube de Braun

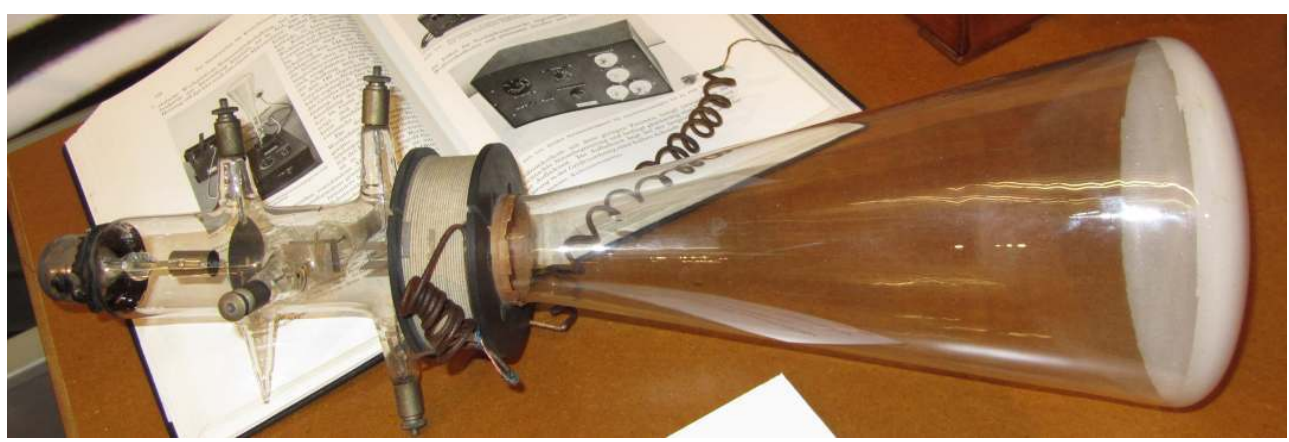

14 C'est la naissance de l'enregistreur cathodique : le premier est sans doute celui de Dufour ${ }^{12}$; il permet de gagner un facteur 100 dans les fréquences d'enregistrement (ENS 1914-1923). Durant un siècle, le tube cathodique aura une belle descendance: oscilloscopes enregistreurs et télévision. 
Désormais, qu'elles soient mécaniques, électromagnétiques ou cathodiques, les techniques d'enregistrement oscillographiques analogiques vont être appliquées à tous les domaines de recherche, scientifiques puis industriels. Rappelons quelques applications majeures, dont le support reste souvent l'argentique :

- 1910 : Hospitalier (EMPCI) conçoit avec Jules Carpentier un manographe adapté à l'étude des moteurs à combustion interne ;

- 1927 : Conrad Schlumberger conçoit et réalise le premier enregistreur de résistivité des sols dans les puits de forage pétroliers, premier élément du formidable essor de cette technique de prospection ;

- 1933 : André Langevin et Paul Beaudouin (ESPCI) associent aux enregistreurs la technique des capteurs de pression piézo-électrique, appliquée à la prospection sismique (CGG 1935) ${ }^{13}$ et à divers domaines.

- 1935-1945 : Henri Gondet, Paul Beaudouin (ESPCI) et François Hussenot (X-SupAero) réalisent les premiers enregistrements d'essais en vol et mettent au point les premières « boites noires » réellement opérationnelles.

\section{Boîte noire enregistreur HB}

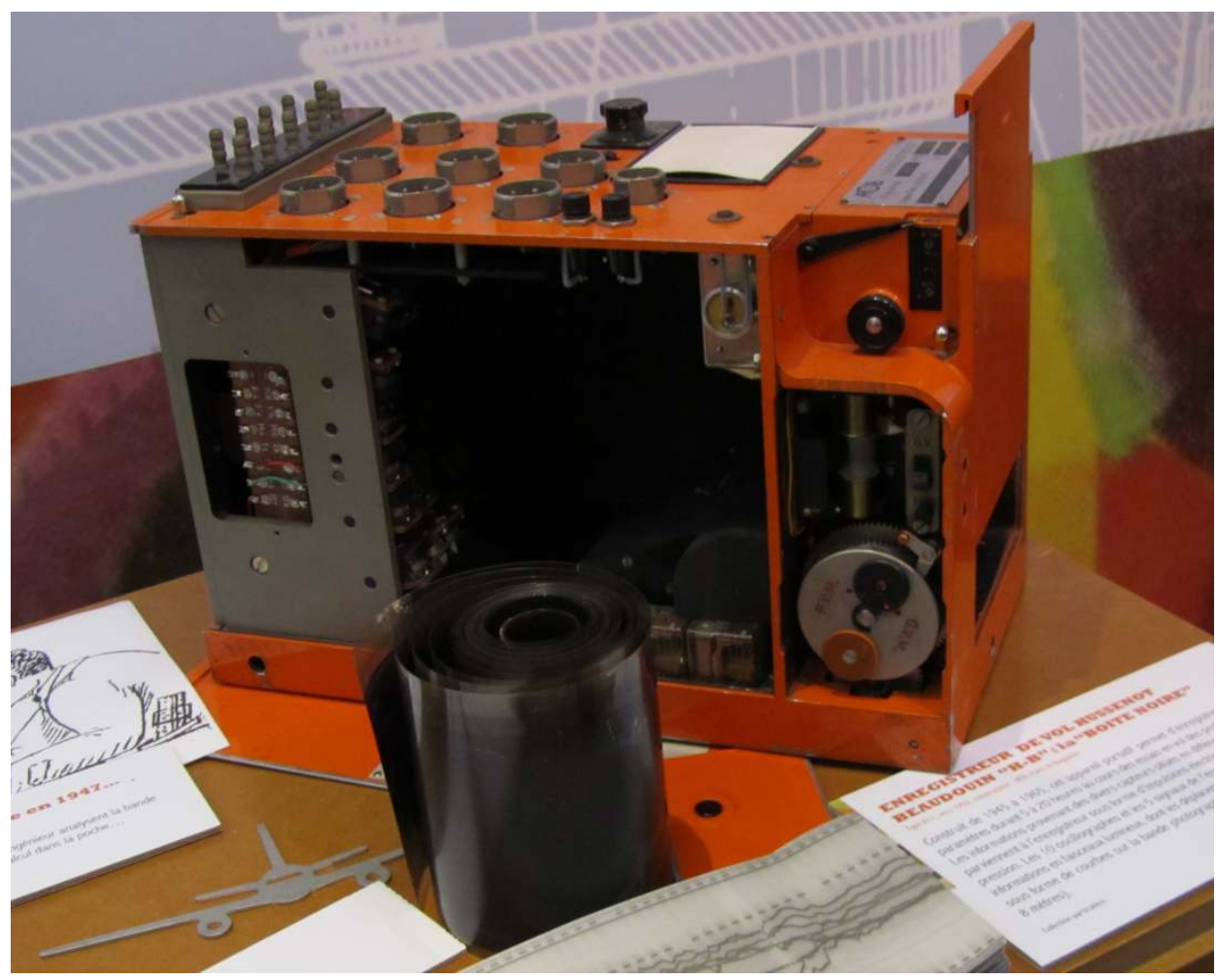

Et il faudra garder dans la mémoire (!) du $\mathrm{xx}^{\mathrm{e}}$ siècle aussi bien les très nombreux enregistreurs classiques et inusables de la Maison Jules Richard que l'extraordinaire développement du cinéma scientifique, initié par Jean Painlevé et Jean Comandon sur les pas de Jules Marey. Reposant sur des principes déjà bien éprouvés mais mis en œuvre avec une technicité croissante, ils permirent découvertes et développements essentiels, tant scientifiques que techniques et pédagogiques. 


\section{Le magnétisme : de l'oreille à l'ordinateur}

Pendant ce temps débute en 1898 une aventure radicalement différente pour l'enregistrement des mesures, lorsqu'est breveté et réalisé par Valdemar Poulsen ${ }^{14}$ l'enregistrement magnétique imaginé dix ans avant par Oberlin Smith ${ }^{15}$. Trois ans après la thèse de Pierre Curie, qui fonde le magnétisme moderne, l'ingénieur danois Poulsen fait défiler un fil d'acier entre les pôles d'un électro-aimant relié à un microphone.

Schéma de l'enregistreur à fil magnétique tel qu'imaginé en 1888 par Oberlin Smith

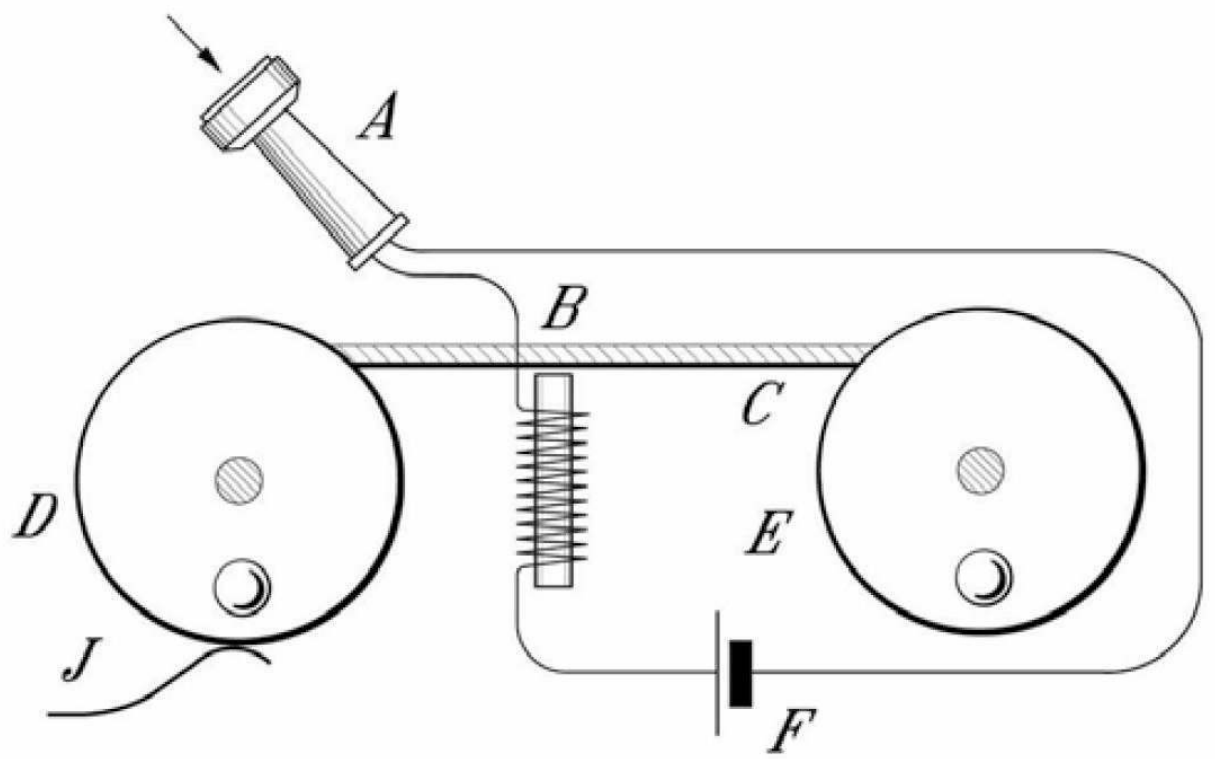

Dans la version brevetée et réalisée dix ans plus tard par Valdemar Poulsen, un électroaimant efface le fil, un second relié à un microphone inscrit l'information, et un troisième la lit dans un écouteur. Tout ceci sans aucune amplification.

L'entrefer d'un second électroaimant est relié à un écouteur téléphonique pour restituer les sons. Plus tard, le fil du "Telegraphon» est remplacé par un ruban d'acier ${ }^{16}$, on amplifie le signal et on accélère la vitesse de défilement. En 1935 une importante amélioration connaitra un succès foudroyant. Il s'agit du procédé «Magnétophon $»^{17}$ : la bande magnétique est constituée de plastique, sur lequel est déposée une poudre d'oxyde de fer. D'autres améliorations et innovations font évoluer ce procédé dans les décennies suivantes, jusqu'à conduire en 1956 au "disque dur », le support de données le plus efficace dont nous disposons aujourd'hui.

\section{De la cassette aux tambours, l'enregistrement numérique}

Par sa souplesse, le support plastique supplantera tous les autres. La "cassette $»^{18}$ lui donnera une dimension populaire dans les années 1960 et jusqu'à la fin des années 1980. Les premiers enregistrements magnétiques ne concernent que le son, mais, durant la $\mathrm{S}$ econde Guerre mondiale et dans les années 1950, une série d'avancées ouvre la voie à l'enregistrement numérique de données. 


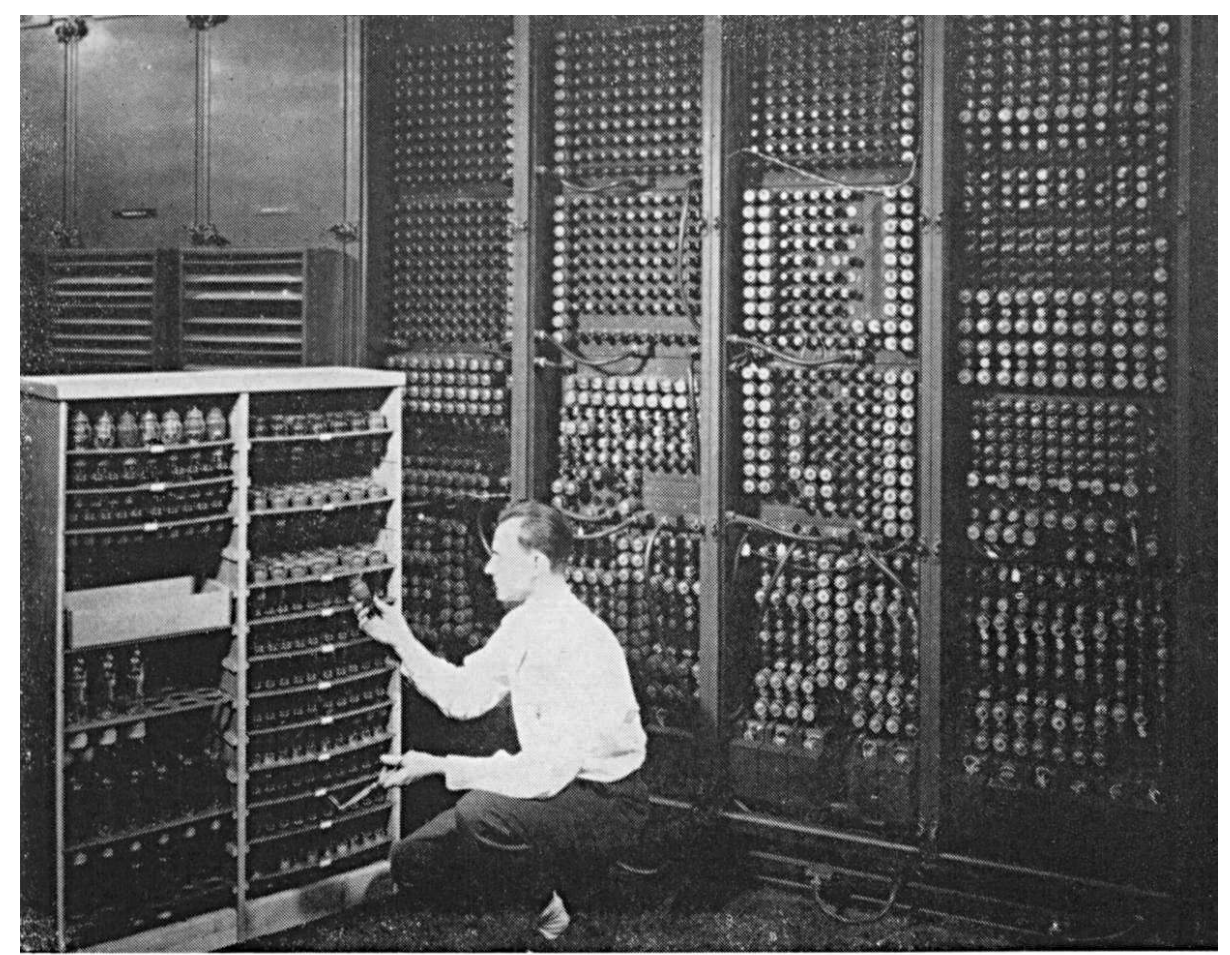

Replacing a bad tube meant checking among ENIAC's 19,000 possibilities.

La première est l'invention de l'ordinateur ${ }^{19}$, initialement destiné à déchiffrer les communications militaires nazies. Début 1946, l'ENIAC (Electronic Numerical Integrator and Computer) est le premier ordinateur entièrement électronique. Utilisant 17468 tubes, il pèse plus de 30 tonnes, occupe $72 \mathrm{~m}^{2}$ et consomme $160 \mathrm{~kW}$. Il ne peut fonctionner que quelques heures avant qu'un tube ne "grille». Il fallait alors chercher pendant des heures le tube responsable...

21 Ce gigantisme disparaît à partir des années 1950, en raison de l'invention du transistor ${ }^{20}$ (1947) et du stockage magnétique sur de nouveaux supports, d'abord sur des « tambours » cylindriques, puis sur des disques durs magnétiques (IBM, 1956) ${ }^{21}$.

\section{Le stockage magnétique tient la corde, et pour longtemps}

22 En un demi-siècle, les disques durs (HDD pour Hard Disk Drive) ont vu leurs performances (en termes de bits enregistrés $/ \mathrm{cm}^{3} / \mathrm{s}$ ) multipliées par mille milliards $\left(10^{12}\right)$ (figure 1$)$. 


\section{Information traitée et/ou enregistrée / seconde $/ \mathrm{cm}^{3}$}

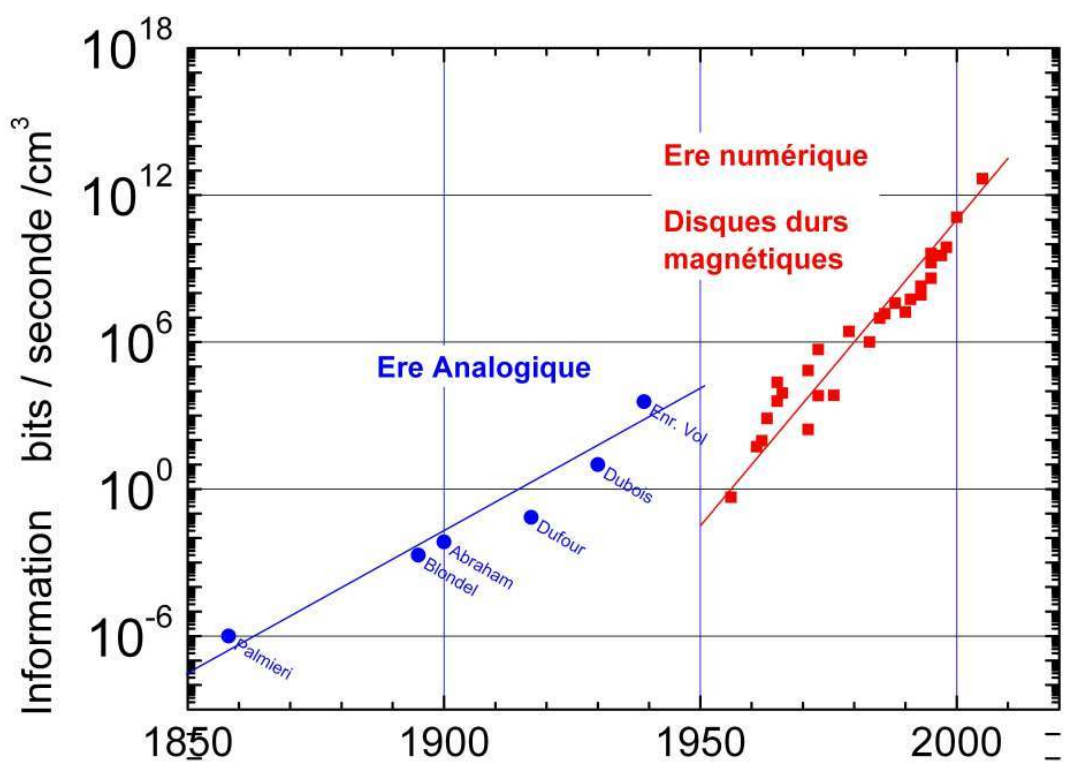

PERFORMANCES HDD. L'INTÉgRATION À gRANDE ÉCHELLE -UN MICROPROCESSEUR COMPORTE AUJOURD'HUI PLUSIEURS MILLIARDS DE TRANSISTORS-, LA MINIATURISATION DES SUPPORTS D'ENREgISTREMENT, PUIS LA MISE EN œUVRE DE L'INTERNET, BOUSCULENT RADICALEMENT LE TRAITEMENT DE TOUS LES TYPES D'INFORMATION, AINSI QUE LEURS SUPPORTS, LEURS FLUX, LEURS ARCHIVAgE : AU COURS DU XXE SIÈCLE LA RAPIDITÉ DE TRAITEMENT ET D'ENREgISTREMENT DE L'INFORMATION PAR UNITÉ DE VOLUME ET PAR SECONDE, A AUgMENTÉ D'UN FACTEUR DIX MILLE MILLIARDS, SOIT 1013 ! 1968 , mémoires à bulles en 1970, n'ont été commercialisés que quelques années avant d'être rapidement dépassés par les progrès des disques durs. À partir des années 1990, la magnétorésistance géante ${ }^{26}$ accélère encore la progression de l'enregistrement magnétique pour des décennies. Notons que l'enregistrement numérique de données sur 
bande magnétique est pourtant toujours utilisé pour sa robustesse, sa compacité et son faible coût. Son défaut essentiel reste son temps d'accès relativement long, ce qui a peu d'inconvénients dans une fonction d'archivage à long terme.

\section{Et demain ?}

27 L'inventivité des hommes est telle que de très nombreuses technologies sont confrontées en une compétition quasi darwinienne. Après l'apparition d'une grande diversité d'enregistrements "pour l'œil», les méthodes «pour l'ordinateur» installent progressivement après 1960 un standard, et une technologie dominante. Le standard numérique : l'information est mise sous forme binaire, facilement stockable, traitable, et parfaitement reproductible. La technologie dominante: le disque dur magnétique. L'efficacité du numérique est telle, qu'aucun type d'information n'échappe plus à ces technologies, qu'elle soit scientifique - sa destination première -, technique, artistique ou culturelle.

Si la progression des performances de calcul des microprocesseurs devrait bientôt s'infléchir - vers 2040 un transistor aurait la taille d'un atome à la vitesse où progressent les technologies - il n'en est pas de même pour les technologies d'enregistrement magnétique qui sont encore loin de leurs limites physiques. Elles auront des prolongements nanométriques particulièrement performants durant les prochaines décennies ${ }^{27}$.

L'empreinte de l'espèce humaine sur la terre est telle depuis deux siècles, que l'on a pu nommer cette période si courte à l'échelle des temps géologiques, mais particulièrement décisive, l'Anthropocène ${ }^{28}$. En 2010, des chercheurs ont créé une bactérie dont le génome a été entièrement synthétisé par des méthodes chimiques ${ }^{29}$. Ce premier être vivant dont le parent est un "simple ordinateur ", marque une étape essentielle du traitement de l'information biologique dans les «mémoires externes » créées par homo sapiens sapiens. Serait-ce un moment fort de l'Anthropocène?

\section{NOTES}

1. Les techniques actuelles de neurophysiologie et d'imagerie telles que l'IRM confirment le rôle essentiel de la mémoire dans des tâches telles que jouer aux échecs : les experts agissent la plupart du temps par "réflexe », voir Xiaohong Wan et al., "The Neural Basis of Intuitive Best Next-Move Generation in Board Game Experts », Science, vol. 331, n 6015, p. 341-346, janvier 2011, p. 341-346.

2. Bruno Jacomi, «L'Anémomètre des Pajot d'Ons-en-Bray ", La Revue, Musée des Arts et Métiers, $\mathrm{n}^{\circ} 30$, juin 2000 .

3. Alexandre Herlea, "Les indicateurs de pression, leur évolution en France au XIX ${ }^{\mathrm{e}}$ siècle ", in Christine Blondel, Françoise Parot, Anthony Turnur et Mari Williams (éd.), Studies in the History of Scientific Instruments, papers presented at the 7th Symposium of the Scientific Instruments Commission of the Union Internationale d'Histoire des Sciences, Paris 15-19 September 1987, Londres, Rogers Turner 
Books, pour le Centre de Recherche en Histoire des Sciences et des Techniques de la Cité des Sciences et de l'Industrie, 1989.

4. Laurent Mannoni, "Le sphygmographe, une invention en trois étapes ", Thierry Lefebvre, Jacques Malthete et Laurent Mannoni (dir.), Sur les pas de Marey, Sciences et cinéma, Paris, L'Harmattan/SEMIA, 2004.

5. Serge Benoit, Daniel Blouin, Jean-Yves Dupont, Gérard Emptoz, «Chronique d'une invention : le phonautographe d'Edouard-Léon Scott de Martinville et les cercles parisiens de la science et de la technique ", Documents pour l'histoire des techniques, $\mathrm{n}^{\circ} 17,1^{\mathrm{er}}$ semestre 2009, Paris, CDHTECNAM.

6. Stéphane Le Gars, "Image et mesure: deux cultures aux origines de l'astrophysique française ", Cahiers François Viète, $n^{\circ} 11-12$, Centre François Viète, Epistémologie, Histoire des sciences et des Techniques, Université de Nantes, 2006.

7. Voir les publications de l'INGV (Instituto nazionale di geofisica et vulcanologia) à Naples, Antonio Nazzaro, Bruno Tramma, Lorenzo Casertano Magda de Lucia (www.ov.ingv.it).

8. Léon Regray, « Notice sur le Wagon d'expériences ", C ${ }^{\text {ie }}$ des Chemins de Fer de l'Est, Paris, mai 1878.

9. Charles Fabry, «Henri Abraham. Mort pour la France (1868-1944)», Cahiers de Physique, n 29-30, juin 1947, p. 3-24.

10. André Blondel, « Oscillographe », La Lumière Électrique, t. XLI, $\mathrm{n}^{\circ} 35$, août 1891, C. R. Académie des Sciences, 1893 et 1899.

11. André Blondel: "Sur l'inscription directe des courants variables ", Congrès des Électriciens, Paris, 1900, C. R. Académie des Sciences, 1900, extrait du volume consacré à l'Exposition de 1900, p 264-295.

12. A. Dufour, L'Oscillographe cathodique, Paris, Chiron, 1923.

13. Pierre Hémardinquer, «Les nouvelles applications du quartz piézo-électrique », La Nature, CNAM-CNUM, 1935, p. 215-219.

14. Valdemar Poulsen, «(en) Brevet U.S. 661619 Method of Recordings and Reproducing Sounds or Signals », Magnetic Tape Recorder. Mentionnons les travaux de Bernard Teston (UMR 6057, Université Aix-Marseille) sur le signal de parole et son enregistrement, en particulier «À la poursuite du signal de la parole », Actes des journées d'études sur la parole 26 JEP, 7-10, 2006.

15. Oberlin Smith, "Some Possible Forms of Phonograph», The Electrical World, September 8, 1888 , p. 116.

16. En 1920-1930, Fritz Pfleumer et Kurt Stille améliorent le procédé Poulsen en passant du fil au ruban d'acier puis surtout à la bande de papier ou de plastique enduite d'oxydes de fer aimantables. En 1928, Fritz Pfleumer dépose le brevet de la première bande magnétique.

La BBC, créée en 1931, utilise un «blattnerphone » qui pèse près d'une tonne : les émissions sont enregistrées sur des bandes d'acier qui défilent à une vitesse de l'ordre de 1 mètre par seconde. Le blattnerphone sera utilisé jusqu'en 1945.

17. En 1935, AEG fabrique le premier appareil d'enregistrement magnétique à bande plastique, tandis que BASF met au point la première bande magnétique en acétate de 6,3 millimètres de large commercialisable. Il devient possible, grâce au « Magnetophon » à piles, d'enregistrer le son en direct en tous lieux.

18. En 1961, Philips met au point la mini-cassette audio.

19. Pierre-Éric Mounier-Kuhn, L'Informatique en France de la Seconde Guerre mondiale au Plan Calcul. L'émergence d'une science, Paris, Presses universitaires Paris-Sorbonne, 2010.

20. Le transistor a été inventé en décembre 1947 par John Bardeen, William Shockley et Walter Brattain, de la compagnie Bell Téléphone, qui ont reçu le prix Nobel de physique en 1956.

21. En 1956, le RAMAC 305 (pour Random Access Method of Accounting and Control) a été présenté par IBM. Il pesait plus d'une tonne et utilisait 50 disques en métal de $60 \mathrm{~cm}$ de diamètre, tournant à $1200 \mathrm{tr} / \mathrm{min}$, comportant 100 pistes par face. Deux têtes de lecture/écriture 
pouvaient se déplacer d'un plateau à un autre en moins d'une seconde. Sa capacité était de 5 Mo et le débit de 8,8 kilo-octets/s.

22. Les supports à lecture optique du type CD ont été inventés conjointement en 1979 par les firmes Philips et Sony avec la participation de Hitachi. Selon les rumeurs, la capacité du CD 12 centimètres (650 Moctet) a été augmentée à la demande de Herbert von Karajan, pour que la Neuvième symphonie de Beethoven tienne sur un seul CD...

23. Le HVD (Holographic Versatile Disc) stocke les données dans un hologramme numérique. Les disques HVD ont une capacité de stockage maximale de 3,9 téraoctets. Le HVD est lu par deux faisceaux laser, vert et rouge, qui lisent les données par interférence.

24. La mémoire flash utilise des transistors MOS, où l'information est stockée dans une "grille flottante » au milieu de l'oxyde entre le canal et la grille. Les données sont conservées lorsque l'alimentation électrique est coupée.

25. Mark H. Kryder et Chang Soo Kim, "After Hard Drives - What Comes Next?», IEEE Transactions on Magnetics, vol. 45, $\mathrm{n}^{\circ}$ 10, octobre 2009.

26. Albert Fert et Peter Grünberg ont reçu le Prix Nobel de Physique 2007 «for the discovery of Giant Magnetoresistance ".

27. Stuart S. P. Parkin, et al., "Magnetic Domain-Wall Racetrack Memory ", Science, avril 2008, vol. 320, n 5873, p. 190-194; cet article s'appuie sur plusieurs brevets déposés entre 2004 et 2007.

28. Claude Lorius, Laurent Carpentier, Voyage dans l'Anthropocène, Paris, Actes Sud, 2010.

29. Daniel G. Gibson et al., "Creation of a Bacterial Cell Controlled by a Chemically Synthesized Genome », Science, vol. 329, n 5987, mai 2010, p. 52-56.

\section{RÉSUMÉS}

Ce texte présente l'exposition «Enregistrer pour Comprendre (Espace Pierre-Gilles de Gennes de l'ESPCI, octobre 2010- juillet 2011) qui retrace l'histoire des « mémoires externes » inventées par l'homme, de la gravure sur pierre à l'ordinateur.

This paper presents the exhibition «Enregistrer pour Comprendre " ( Recording and Understanding », Espace Pierre-Gilles de Gennes, ESPCI, october 2010 - july 2011), which traces the history of « external memories » invented by mankind, from stone carving to computers.

\section{INDEX}

Mots-clés : enregistrement, instruments de mesure, histoire des sciences et des techniques

Keywords : recording, measuring instruments, history of sciences and technologies 


\section{AUTEURS}

\section{DENIS BEAUDOUIN}

Espace Pierre-Gilles de Gennes de l'ESPCI ParisTech

Parmi les publications : Denis Beaudouin, Charles Beaudouin : une histoire d'instruments scientifiques, EDP Sciences, Les Ulis, 2005.

\section{MICHEL LAGUËS}

Espace Pierre-Gilles de Gennes de l'ESPCI ParisTech

Parmi les publications : Michel Laguës, Invariances d'échelle. Des changements d'état à la turbulence (avec Annick Lesne), Paris, Belin, 2003. 